\title{
Analysis of Antigens of Rhodococcus Species by Rocket Immunoelectrophoresis
}

\author{
SOTIROS D. CHAPARAS, ${ }^{1}$ MICAH I. KRICHEVSKY ${ }^{2}$ FRANCES A. BENEDICT, ${ }^{3}$ GEORGE C. \\ ROYAL, ${ }^{4}$ AND IRIS S. HYMAN ${ }^{1}$ \\ Bureau of Biologics, Food and Drug Administration, Bethesda, Maryland $20205 ;^{1}$ Division of Intramural \\ Research, National Institutes of Health, Bethesda, Maryland 20205; Executive Directorate of Regional \\ Operations, Food and Drug Administration, Rockville, Maryland $20852 ;^{3}$ and Department of Microbiology, \\ School of Medicine, Howard University, Washington, D.C. $20059^{4}$
}

\begin{abstract}
Seventeen representative strains of Rhodococcus and related taxa were analyzed for antigenic relationships by rocket immunoelectrophoresis. A good correlation was found between data from this form of analysis and previously reported numerical phenetic and deoxyribonucleic acid reassociation data. Rhodococcus ruber and Rhodococcus erythropolis strains formed distinct clusters in a dendrogram produced by average linkage analyses for dissimilarity matrices. Rhodococcus equi, Nocardia asteroides, and an unidentified Rhodococcus strain formed single-member clusters, whereas Rhodococcus coprophilus was found to be heterogeneous. In contrast, $R$. erythropolis and $R$. ruber were found to be distinct species. The discrepancies between deoxyribonucleic acid pairing and rocket immunoelectrophoresis results can be explained on the basis of suboptimal antigen or antiserum preparations.
\end{abstract}

The genus Rhodococcus was redefined $(6,13)$ to include some organisms of the "rhodochrous" complex which formed a heterogeneous cluster that separated distinctly from aggregate phena corresponding to the genera Mycobacterium and Nocardia. Goodfellow and Alderson (6) recognized 10 species in the genus, including the type species, Rhodococcus rhodochrous. In studies of antigenic relationships among species of Mycobacterium $(2,3)$, the fused rocket immunoelectrophoretic procedure was found to be a valuable adjunct in confirming suggested taxonomic relationships. In this study we applied this procedure to an analysis of antigens in selected Rhodococcus strains.

\section{MATERIALS AND METHODS}

Bacterial strains. The strains studied (Table 1) were received from Michael Goodfellow and have been described previously $(5,7)$.

Antigen preparation. Primary cultivation was on oleic acid albumin and Lowenstein-Jensen egg yolk slants for 2 to 4 days, after which cells were inoculated into Blake bottles containing Long synthetic medium, harvested, and sonicated as described previously (7). Immunological studies were performed with centrifuged supernatants.

Production of antibody. Rabbits were immunized with a Freund-type water-in-oil emulsion consisting of an aqueous phase of sonic extract and an equal volume of Arlacel A-Drakeol 6VR (35:65) containing $10 \mathrm{mg}$ of dried organisms per $1.0 \mathrm{ml}$. The emulsion was prepared by repeated passage of the mixture through a double-hub needle connected to two $6.0-\mathrm{ml}$ syringes.
Each rabbit received a total of $1.0 \mathrm{ml}$ of an emulsion distributed equally into the four footpads and subcutaneously into six lateral positions. Subsequent injections consisted of $0.5 \mathrm{ml}$ of sonic extract given subcutaneously every 2 weeks in the nuchal area until precipitin production appeared to be maximal.

Fused rocket immunoelectrophoresis. We used a modification of the fused rocket immunoelectrophoresis procedure of Svendsen (12), as described previously $(2,3)$; antigen was placed in overlapping oval antigen wells and was forced electrophoretically into an antibody-containing region of Veronal-buffered agar ( $\mathrm{pH} 8.6$; ionic strength, $0.075 \mathrm{M}$ ). Electrophoresis was performed for $5 \mathrm{~h}$ at $2.7 \mathrm{~V} / \mathrm{cm}$ and $10 \mathrm{~mA}$.

Antigenic and computer analyses. Precipitin bands developed with homologous and heterologous antigen preparations by means of the fused rocket immunoelectrophoresis procedures were analyzed by the percent qualitative sharing (PQS) method (2), as follows: $\mathrm{PQS}=$ (number of bands with heterologous preparation)/(number of bands with homologous preparation) $\times 100$. This method takes into account whether antigenic determinants are present and weighs weak bands as much as intense bands.

The data in Table 2 were examined by cluster analysis via C-lab (maintained by the Division of Computer Research and Technology, National Institutes of Health, Bethesda, Md.). The values were entered into a 17-by-17 matrix. The values within the lower triangle formed by the homologous diagonal of values were read into another file which contained the similarity triangle matrix. C-lab was written to accept dissimilarity matrices; thus, the similarity matrix was converted into a dissimilarity matrix. The dendrogram prepared for dissimilarity matrices (see Fig. 1) was based on average linkage. The distance of each point from every other point was calculated, and clusters 
TABLE 1. Strains used in this study

\begin{tabular}{|c|c|c|}
\hline Strain & Species & $\begin{array}{l}\text { No. and cluster of } \\
\text { Goodfellow and } \\
\text { Alderson }\end{array}$ \\
\hline 1 & R. ruber & $\mathrm{N} 324 / 1 \mathrm{E}$ \\
\hline 2 & R. ruber & $\mathrm{N} 325 / 1 \mathrm{E}$ \\
\hline 3 & R. ruber & N361/1D \\
\hline 4 & R. ruber & $\mathrm{N} 420 / 1 \mathrm{D}$ \\
\hline 5 & R. coprophilus & $\begin{array}{l}\mathrm{N} 650 / R \text {. ruber- } \\
\quad R . \text { coprophilus }\end{array}$ \\
\hline 6 & R. coprophilus & N651/1J \\
\hline 7 & R. equi & N652 R. equi \\
\hline 8 & R. erythropolis & $\mathrm{N} 38 / 1 \mathrm{H}$ \\
\hline 9 & $R$. erythropolis & $\mathrm{N} 53 / 1 \mathrm{H}$ \\
\hline 10 & $R$. erythropolis & N41 \\
\hline 11 & R. rhodochrous & $\mathrm{N} 5 / 1 \mathrm{~A}$ \\
\hline 12 & $R$. rhodochrous & $\mathrm{N} 54 / 1 \mathrm{~A}$ \\
\hline 13 & Rhodococcus species & $\mathrm{N} 124^{b} / 14 \mathrm{E}$ \\
\hline 14 & R. coprophilus & N653 ${ }^{\circ}$ \\
\hline 15 & R. corallinus & $\mathrm{N} 655 / 1 \mathrm{C}$ \\
\hline 16 & $N$. asteroides & N99 \\
\hline 17 & R. terrae & $\mathrm{N} 656 / 1 \mathrm{C}$ \\
\hline
\end{tabular}

${ }^{a}$ See reference 6 .

${ }^{b}$ Not assigned to any species.

${ }^{c}$ Species identification not clear.

were formed with the closest points. Average distances of proximal clusters were determined.

Skin tests. Guinea pigs were sensitized and skin tested as previously described (1); each subcutaneous injection of a water-in-oil emulsion contained $2 \mathrm{mg}$ of dried organisms. At 6 weeks after sensitization, guinea pigs were skin tested with $0.1 \mathrm{ml}$ of a saline solution containing homologous and heterologous antigen preparations. The dose of each antigen used was predetermined to render a reaction size of about $13 \mathrm{~mm}$ in the homologously sensitized animals.

Specificity differences (SPD) for each reaction were determined by the method of Magnusson (8), as follows: $\mathrm{SPD}=(\mathrm{Aa}+\mathrm{Bb})-(\mathrm{Ab}+\mathrm{Ba})$, where $\mathrm{Aa}$ and $\mathrm{Bb}$ are the diameters of the homologous reactions of two species and $\mathrm{Ab}$ and $\mathrm{Ba}$ are the diameters of the heterologous reactions for the same two species.

\section{RESULTS}

With the strains studied, the number of precipitin bands produced with cell extracts and homologous antisera in fused rocket immunoelectrophoresis varied from 13 to 50, except for Rhodococcus terrae N656 (formerly Gordona terrae), which produced only 7 bands (Table 2). Table 2 also shows the PQS values. A method has not yet been devised to quantitate accurately the antigens in multiple-component systems in which shared epitopes that react in precipitation reactions might be on different types of carrier molecules. Occasionally, the PQS value of a heterologous antigen can exceed or equal $100 \%$ due either to a close similarity of species or differences in the potencies of antigen preparations or both. For computer analyses, one value of $107 \%$ was reduced to $100 \%$.

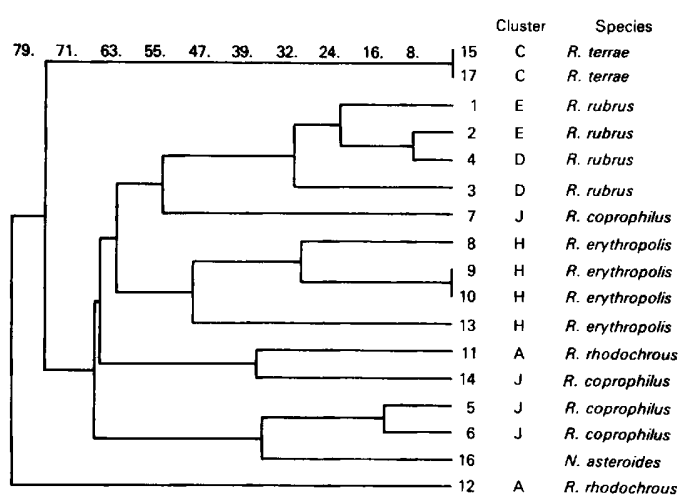

FIG. 1. Average linkage for 16 strains of Rhodococcus and 1 strain of $N$. asteroides.

The dendrogram in Fig. 1 illustrates the average linkage values for the 17 strains studied. It also demonstrates the general relationship of most of the organisms. All of the organisms defined by Goodfellow and Alderson (6) as Rhodococcus ruber formed a closely related cluster as determined by precipitin analysis and were clearly separated from the three clustered Rhodococcus erythropolis strains. Two of the three Rhodococcus coprophilus strains appeared to be very closely related, whereas the other strain (strain N653) was less related. The $R$. terrae strain appeared to be closely related to Rhodococcus corallinus on the dendrogram, but the $R$. rhodochrous strains were separated from one another. The single Nocardia asteroides strain examined appeared to have affinity to $R$. coprophilus $\mathrm{N} 650$ and N651; the reason for this apparent discrepancy is discussed below.

Because of biological variation both in the preparation of sonic extracts of organisms and in the antibody responses of rabbits, the PQS values of any two related species may be disparate (Table 2). Some of the discrepancies between two species were lessened when values were averaged to obtain the reciprocal average PQS (RAP), as follows: RAP $=(\mathrm{PQS}$ of A with serum $\mathrm{B}+\mathrm{PQS}$ of $\mathrm{B}$ with serum A)/2. The RAP values are shown in Table 3 , together with the corresponding skin test SPDs (see above).

In general, there was good agreement between the results obtained by skin testing and those obtained by antigen analysis; discrepancies were observed in only 27 of 136 heterologous values (Table 3), either when a low SPD $(<3 \mathrm{~mm})$ was accompanied by an RAP of $55 \%$ or less or when an SPD of $>3 \mathrm{~mm}$ corresponded to an RAP value greater than $55 \%$. Possible reasons for these discrepancies are discussed below.

\section{DISCUSSION}

Analyses of precipitinogens detectable with homologous and heterologous antisera are sub- 


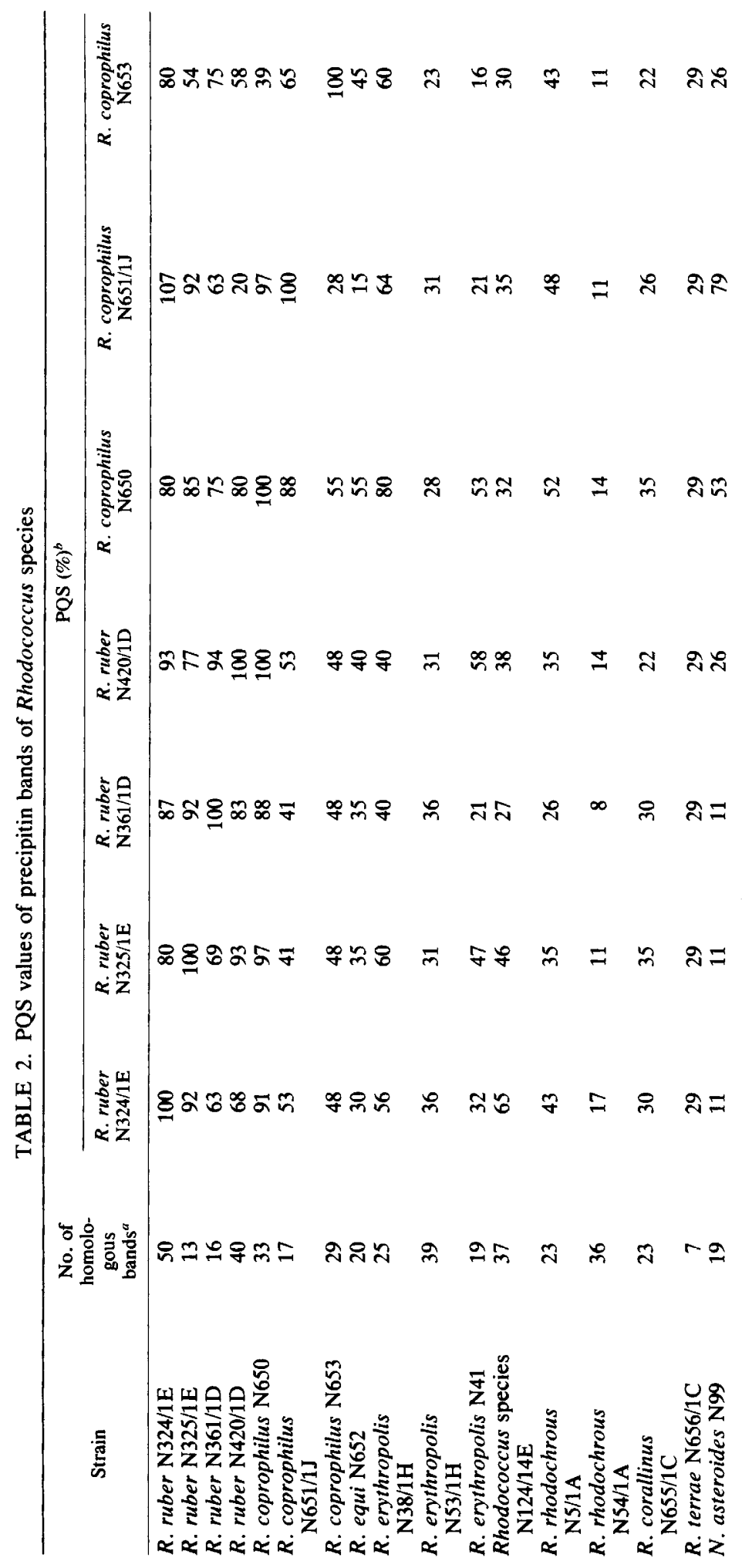




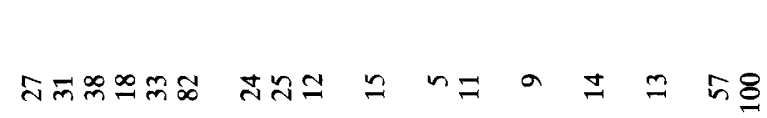

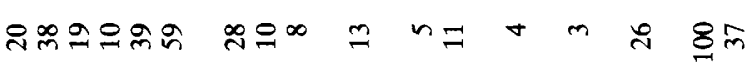

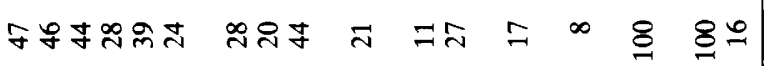

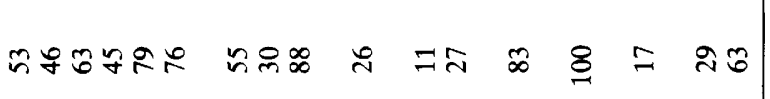

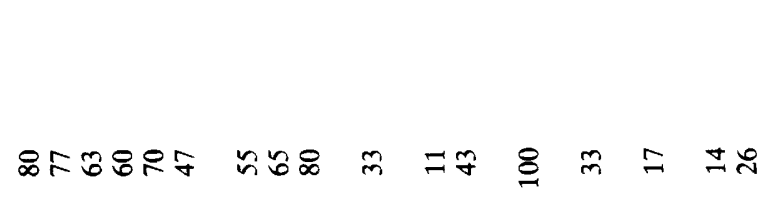

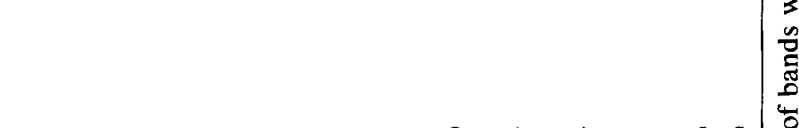
ธำำกำกำ

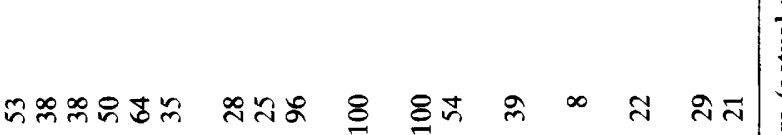

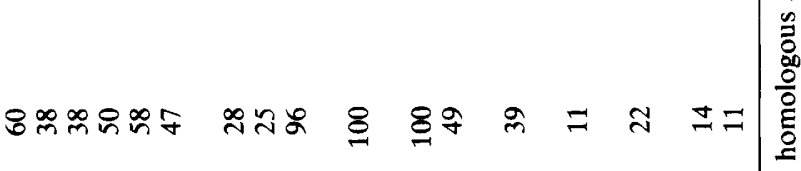

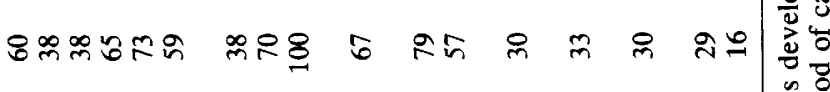

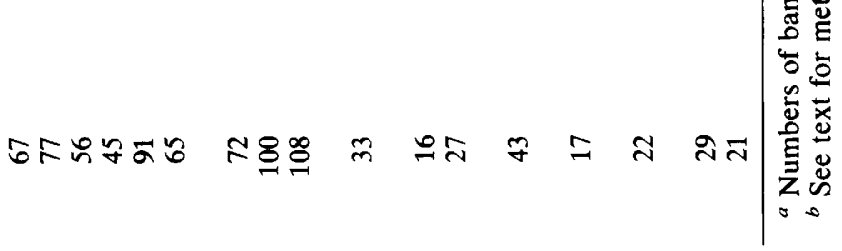


292 CHAPARAS ET AL.

INT. J. Syst. BACTERIOL.

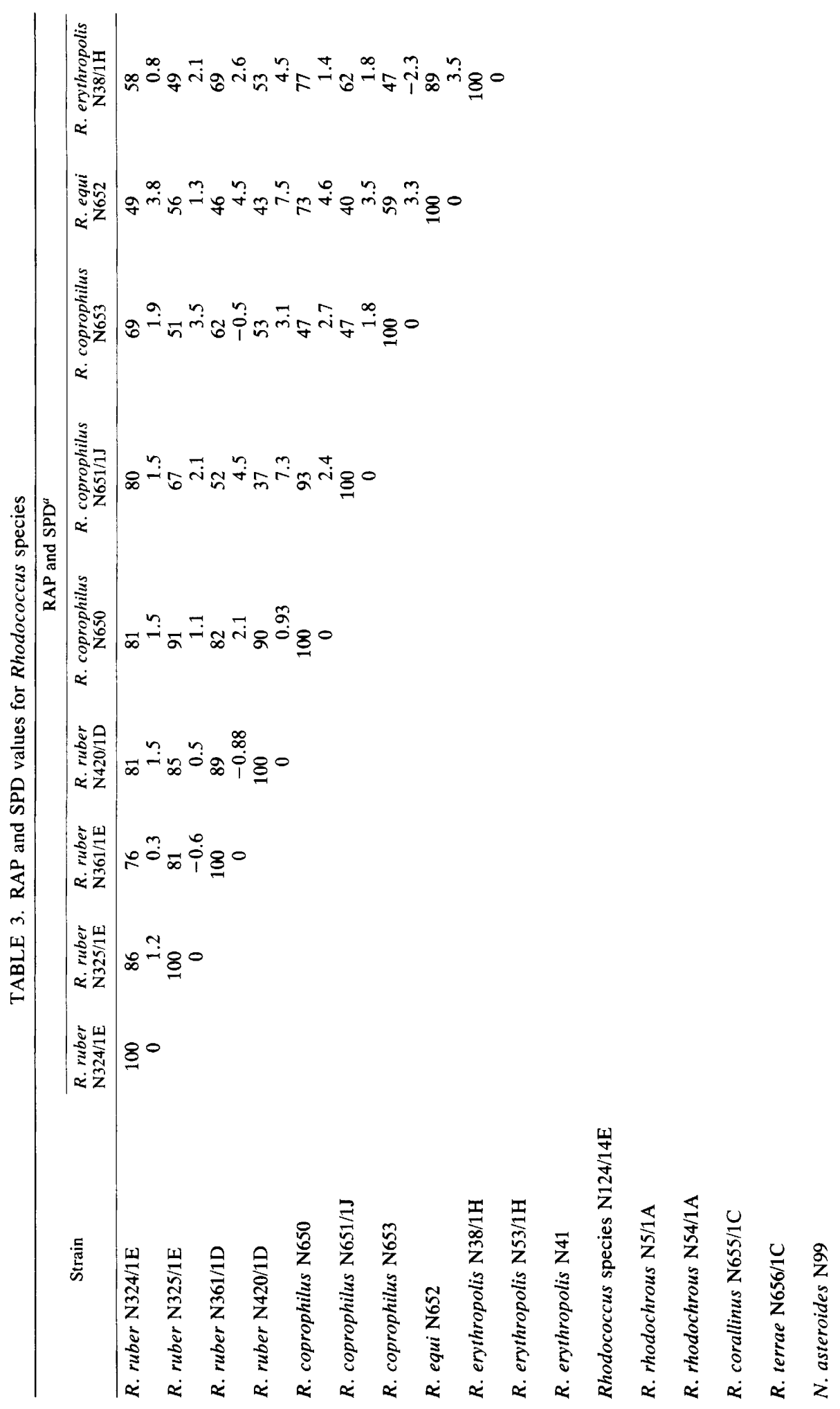


VoL. 32, 1982

ANTIGENS OF RHODOCOCCUS SPECIES

293

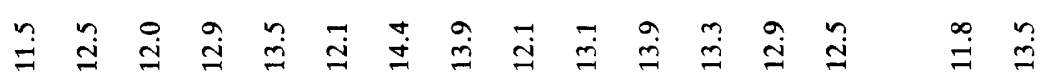

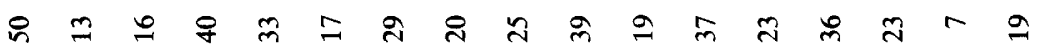

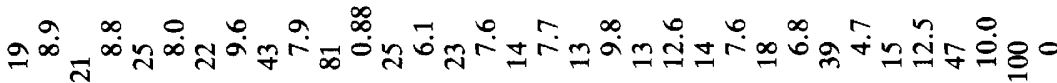

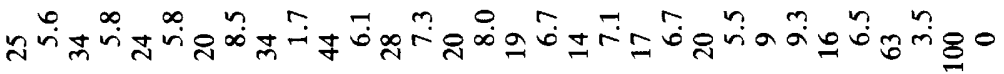

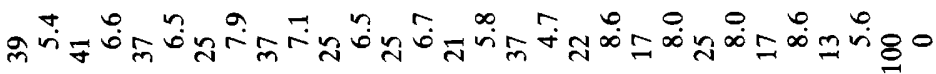

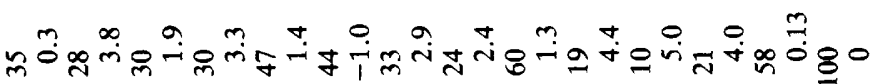

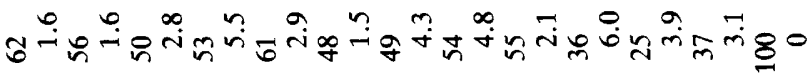

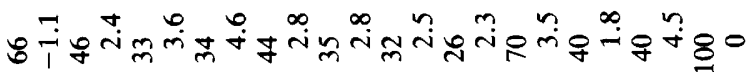

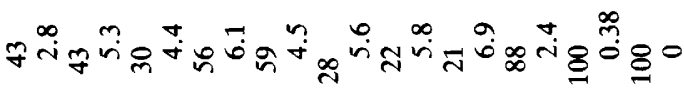

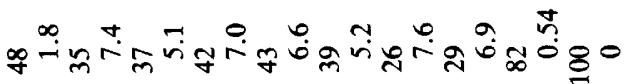

$$
\begin{aligned}
& \text { |0 }
\end{aligned}
$$


ject to variations in the antigenic preparations and in the immune responses. Despite such variations, the procedures described above offer a powerful means for evaluating taxonomic relationships revealed in numerical phenetic analyses. Theoretically, if the maximum number of antigens could be prepared consistently for all preparations and the maximum humoral and cell-mediated immune responses were induced for each antigen, it should be possible to derive optimal relationships between species by this procedure. Realistically however, we must settle for less. In fact, potent heterologous antigen preparations may produce larger numbers of precipitin bands than less potent homologous antigen preparations. A skin reaction is the summation of a number of reactions induced by specifically sensitized lymphocytes reacting with possibly 50 or more antigens. Despite this, analyses of homologous and heterologous skin test reactions produce results that are very discriminating for species $(4,7,8)$. However, occasionally results may be inconsistent with other taxonomic criteria. This is the case with the relationship between $N$. asteroides $\mathrm{N} 99$ and $R$. coprophilus N651. A close relationship is indicated by the SPD (0.88) (Table 3 ) and by the high RAP value $(81 \%)$. Thus, these two strains appear to be closely related antigenically; however, on the basis of numerical phenetic data they are clearly different (6). The antisera used in the analysis contained from 17 to 19 bands, far from the potentially maximal number of 60 or so. Antibodies may have been raised principally to shared dominant antigens. The potential errors due to antibody preparations are more vividly seen with $R$. corallinus $\mathrm{N} 655$ and $R$. terrae N656. Mordarski et al. (11) found little deoxyribonucleic acid homology between strains of these two species, but in our analyses a high degree of relatedness was noted (Fig. 1). However, this apparent relationship can be explained by the poor antiserum for $R$. terrae (Table 2); only seven precipitin bands were produced, and all were shared with $R$. corallinus. A truer relationship is seen with $R$. corallinus antiserum, which produced 23 bands, only $26 \%$ of which were shared. From Table 2 it is clear that $R$. corallinus is a distinct entity. In a recent study (9), $R$. corallinus and Rhodococcus rubropertinctus formed a single deoxyribonucleic acid homology group, and the former name was reduced in status to a synonym of the latter. An advantage of analyses by precipitation in gels is that individual precipitating antigens can be detected and related to a homologous or heterologous system. Sharing of a high percentage of bands generally reflects a close relationship. However, a poor antiserum or antigen preparation can produce misleading results. In skin testing it is possible that a potent sensitizing skin-reactive component present in a relatively high concentration may dominate an observed skin reaction.

$R$. ruber and $R$. erythropolis each formed distinct clusters (Fig. 1), a result consistent with both numerical phenetic and deoxyribonucleic acid pairing data (9). Rhodococcus strain N124 did not fit clearly into any of the clusters recovered by Goodfellow and Alderson (6) and did not cluster clearly in this study; this strain has yet to be identified to species. Rhodococcus equi also clustered by itself in the dendrogram (Fig. 1).

Mordarski et al. (11) found that much of the deoxyribonucleic acid of $R$. rhodochrous $\mathrm{N} 54$ is homologous with that of strain N5. Although these two strains did not appear to be closely related in the dendrogram based on skin test analyses (Table 3), they appeared to be closely related, as suggested by a low SPD. Antiserum for strain N54 was one of the best antisera in that in rocket immunoelectrophoresis it produced 36 bands with the homologous system. Most of these bands appeared to be strain specific and reduced the PQS value for strain N5. Thus, excellent antisera and antigen preparations can be as misleading as poor ones.

Overall, the skin testing and precipitating procedures which we used were effective in determining the relationships of the species in the genus Rhodococcus, and the results obtained were generally consistent with and confirmed the results of Goodfellow and Alderson (6). Of importance is the production of good quality antisera and antigen preparations in reference quantities for each of the type species. Such reagents should be useful in the classification and identification of bacteria.

\section{ACKNOWLEDGMENT}

We gratefully acknowledge Michael Goodfellow for review and helpful criticisms of this paper.

\section{REPRINT REQUESTS}

Address reprint requests to: Dr. Sotiros D. Chaparas, Bureau of Biologics, Food and Drug Administration, Bethesda, MD 20205.

\section{LITERATURE CITED}

1. Chaparas, S. D., and H. Baer. 1963. The immunology and chemistry of tuberculin. II. Chromatography with Sephadex of the nondialyzable tuberculin-active constituents of BCG culture filtrate. Am. Rev. Respir. Dis, 89:41-48.

2. Chaparas, S. D., T. M. Brown, and I. S. Hyman. 1978. Antigenic relationships among species of Mycobacterium studied by fused rocket immunoelectrophoresis. Int. J. Syst. Bacteriol. 28:547-560.

3. Chaparas, S. D., T. M. Brown, and I. S. Hyman. 1978 Antigenic relationship of various mycobacterial species with Mycobacterium tuberculosis. Am. Rev. Respir. Dis. 117:1091-1097.

4. Chaparas, S. D., and C. J. Maloney. 1978. An analysis of cross reactions among mycobacteria by in vivo and in 
vitro assays of cellular hypersensitivity. Am. Rev. Respir. Dis. 117:897-902.

5. Goodfellow, M. 1971. Numerical taxonomy of some nocardioform bacteria. J. Gen. Microbiol. 64:33-80.

6. Goodfellow, M., and G. Alderson. 1977. The actinomycete genus Rhodococcus: a home for the 'rhodochrous' complex. J. Gen. Microbiol. 100:99-122.

7. Hyman, I. S., and S. D. Chaparas. 1977. A comparative study of the 'rhodochrous' complex and related taxa by delayed type of skin reactions on guinea pigs and by polyacrylamide gel electrophoresis. J. Gen. Microbiol. 100:363-371.

8. Magnusson, M. 1961. Specificity of mycobacterial sensitins. I. Studies in guinea pigs with purified "tuberculin" prepared from mammalian and avian tubercle bacilli, Mycobacterium balnei and other acid fast bacilli. Am. Rev. Respir. Dis. 83:57-65.

9. Mordarski, M., M. Goodfellow, I. Kaszen, A. Tkacz, G.
Pulverer, and K. P. Schaal. 1980. Deoxyribonucleic acid reassociation in the classification of the genus Rhodococcus Zopf 1891 (Approved Lists, 1980). Int. J. Syst. Bacteriol. 30:521-527.

10. Mordarski, M., M. Goodfellow, K. Szyba, G. Pulverer, and A. Tkacz. 1977. Classification of the "rhodochrous" complex and allied taxa based upon deoxyribonucleic acid reassociation. Int. J. Syst. Bacteriol. 27:31-37.

11. Mordarski, M., K. Szyba, G. Pulverer, and M. Goodfellow. 1976. Deoxyribonucleic acid reassociation in the classification of the 'rhodochrous' complex and allied taxa. J. Gen. Microbiol. 94:235-245.

12. Svendsen, P. J. 1973. Fused rocket immunoelectrophoresis. Scand. J. Immunol. 2(Suppl. 1):69-70.

13. Tsukamura, M., S. Mizuno, S. Tsukamura, and J. Tsukamura. 1979. Comprehensive numerical classification of 369 strains of Mycobacterium, Rhodococcus, and Nocardia. Int. J. Syst. Bacteriol. 29:110-129. 